\title{
Near-Infrared Metatronic Nanocircuits by Design
}

\author{
Humeyra Caglayan, ${ }^{1}$ Sung-Hoon Hong,${ }^{1, \dagger}$ Brian Edwards, ${ }^{1}$ Cherie R. Kagan, ${ }^{1,2,3}$ and Nader Engheta ${ }^{1,3,4,5, *}$ \\ ${ }^{1}$ Department of Electrical and Systems Engineering, University of Pennsylvania, Philadelphia, Pennsylvania 19104, USA \\ ${ }^{2}$ Department of Chemistry, University of Pennsylvania, Philadelphia, Pennsylvania 19104, USA \\ ${ }^{3}$ Department of Materials Science and Engineering, University of Pennsylvania, Philadelphia, Pennsylvania 19104, USA \\ ${ }^{4}$ Department of Physics and Astronomy, University of Pennsylvania, Philadelphia, Pennsylvania 19104, USA \\ ${ }^{5}$ Department of Bioengineering, University of Pennsylvania, Philadelphia, Pennsylvania 19104, USA \\ (Received 30 September 2012; revised manuscript received 20 June 2013; published 16 August 2013)
}

Lumped circuit elements (i.e., resistors, capacitors, and inductors) provide the basic building blocks of microelectronic devices ubiquitous in information processing, storage, and communications. The use of these modular quasistatic components can be extended to the nanoscale optical regime to achieve highdensity, high-speed analogues of these traditional circuits. We reimagine these devices in the near-infrared (NIR) regime, making use of a simple nanorod geometry and plasmonic transparent conducting oxides (TCOs). We evaluate their equivalent impedance as lumped circuit elements and construct bandpass and band-stop filters operating at NIR wavelengths. Through variation in the TCO nanorod geometry and the addition of $\mathrm{PbS}$ nanocrystals in between and $\mathrm{NiCr}$ on top of the $\mathrm{TCO}$ nanorods, we present the first designable NIR lumped nanocircuits with tailorable response. The experimental results agree with both circuit models and full-wave simulations.

PACS numbers: 42.70.-a, 78.67.Qa

The ability to modularly arrange circuit elements [e.g., resistors $(R)$, inductors $(L)$, and capacitors $(C)$ ] using wellknown circuit models allows us to design complex microelectronic devices and circuitry for information processing, storage, and communications operating in radio frequency (rf) and microwave domains. These elemental units can be translated to the terahertz, infrared (IR) and visible wavelength regions to achieve high-density, high-speed optical analogues [1-4]. However, it is not possible to achieve this goal by simply reducing the sizes of these elements. The first challenge arises in the innate difficulty of achieving the deeply subwavelength critical dimensions necessary using even state-of-the-art nanoscale fabrication techniques. The second challenge lies in the material dispersion. Indeed, even common materials have very different electric and magnetic responses at $200 \mathrm{THz}$ compared to $200 \mathrm{MHz}$.

It was proposed by Engheta et al., that it is possible to have "lumped" circuit elements at optical frequencies using properly designed and suitably arranged plasmonic and nonplasmonic nanostructures [5-7]. In contrast to conventional electronics in which conduction current is the currency exchanged between devices, these optical devices utilize the "flow" of displacement current which is dominant at optical frequencies [5-8]. By taking advantage of the unique material properties of plasmonic materials at optical frequencies, we can vastly ease the burden on fabrication. This new concept of metamaterial-inspired

Published by the American Physical Society under the terms of the Creative Commons Attribution 3.0 License. Further distribution of this work must maintain attribution to the author(s) and the published article's title, journal citation, and DOI. optical nanocircuitry, dubbed "metatronics", has been applied to various wavelength regimes through a series of theoretical analyses and numerical simulations [7-9]. Furthermore, circuit modeling has been applied to many different applications [10-16]. Recently, experimental verification of nanofilters in the $8-14 \mu \mathrm{m}$ wavelength regime has also been achieved [17]. However, in order to assess possible integration of these optical nanocircuits with silicon photonic devices, we aim to explore and utilize plasmonic materials with low-loss in the NIR regime. In this work, we fabricate and characterize large-area optical NIR filters made of low-loss metatronic nanocircuit elements. We further show the possibility of tailoring the optical response of these filters by changing the impedance values of the nanocircuit elements, following simple rf circuit design equations. We theoretically investigate these filters using full-wave simulations and circuit theory.

In the rf domain, the primary functions of information processing are performed using conventional filters such as bandpass and band-stop filters, realized through suitably arranged resistors, capacitors, and inductors. The simplicity and utility of this filter design make it an ideal assessment for metatronics. Filters functioning at optical frequencies, analogous to rf filters, can be obtained by combining these lumped nanocircuit elements. Within metatronics, the optical material dispersion determines the type of lumped nanostructure, with dielectric materials (with positive real part of permittivity) leading to capacitive behavior and plasmonic materials (with negative real part of permittivity) leading to inductive behavior [5]. By arranging plasmonic and dielectric materials in parallel 
and in series, we realize bandpass and band-stop filters as seen in Figs. 1(a) and 1(b).

While plasmonic materials are readily available at optical frequencies where most metals exhibit a negative real part of permittivity, these metals often suffer from large losses. In contrast, it was recently shown that transparent conductive oxides (TCOs) are promising new candidates as low-loss plasmonic materials in the NIR [18-20]. In this study, we use indium-tin-oxide (ITO). ITO has been widely used as an electrode for displays because of its low electrical resistance and high transmittance in the visible range [20]. However, we are interested in properties in the NIR regime where it is possible to model the permittivity of ITO using the Drude dispersion (see Supplemental Material, Fig. S1 [21]). Using this model we find that the real part of the permittivity is negative with reasonable values for wavelengths longer than $1.3 \mu \mathrm{m}$ and becomes highly negative (i.e., effectively metallic) beyond $13 \mu \mathrm{m}$.

We fabricated arrays of rectangular ITO nanorods (NRs) separated by air gaps on glass substrates using nanoimprint lithography and inductively coupled plasma etching. Figures 1(c) and 1(d) show representative SEM images of fabricated NR array samples. In this NR array design, each constituent plasmonic ITO NR acts as a nanoinductor with parasitic loss (a nanoinductor accompanied by a nanoresistor) and the dielectric air gap region functions as a lossless nanocapacitor.

We found that when these arrays of NRs are illuminated via an incident plane wave, the behavior of the nanocircuits
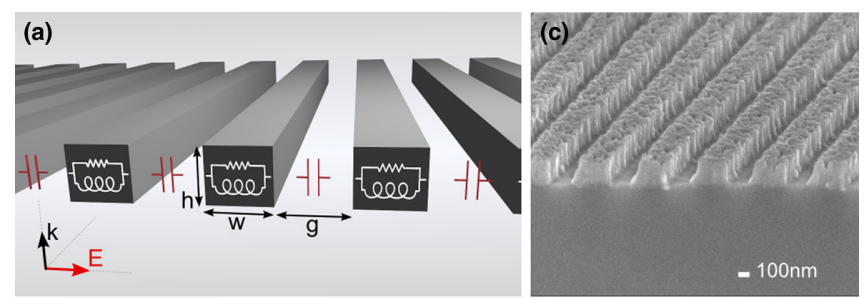

(b)

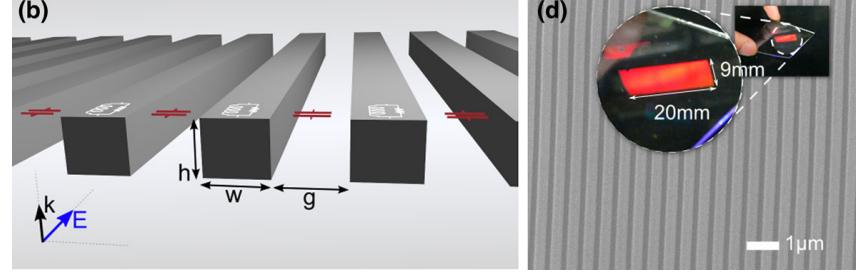

FIG. 1 (color online). Schematic of metatronic nanocircuits. The width and height of the NRs and the width of the air gaps are denoted by $w, h$, and $g$. (a) The $E$ field is perpendicular to the NRs; therefore, nanoinductors (with nanoresistors) and nanocapacitors form a series combination, whereas in (b) the $E$ field is parallel to the NRs, and thus they form a parallel combination of lumped circuit elements. Scanning electron microscopy (SEM) images of a fabricated ITO NR array with $w=320 \mathrm{~nm}, g=180 \mathrm{~nm}$, and $h=150 \mathrm{~nm}$ (c) in cross section and (d) top down (inset: photograph of a NR array over a large area $9 \mathrm{~mm} \times 20 \mathrm{~mm}$ ) is dependent on the polarization of the incident $E$ field, thus making such circuits "stereo circuits". This was also observed in our earlier work in the 8-14 $\mu \mathrm{m}$ regime using an entirely different material [17]. When the $E$-field vector is polarized perpendicular to the rods the arrays act as a "series" $L-C$ combination and indeed behave as band-stop filters. However, when NRs are illuminated by an $E$-field vector parallel to the rods, the arrays function as a "parallel" combination of elements and act as bandpass filters.

Since we have all the necessary circuit elements in our 2D NR array model, we can control the functionality of this metatronic circuit similar to a conventional rf filter: by changing the impedances of the circuit elements. However, in metatronics, this is done by varying the NR width (w) and separation gap (g) to achieve different optical responses. We explore a range of these values and the fabricated sample geometries are listed in Table I as samples A through E.

The transmittance of these samples (A through E) is measured using a spectrophotometer [Figs. 2(a) and 2(b)]. In comparison, we evaluate the transmittance from fullwave simulations [Figs. 2(c) and 2(d)] and we also calculate the transmittance via circuit theory [Figs. 2(e) and 2(f)], fully taking into account the effect of the substrate (See Supplemental Material [21] for the details). In the circuittheory calculations, we used the equivalent impedance of the circuit elements determined by approximating the structure using a simple model based on a uniform electric field. The details of the calculation can be found in Ref. [17]. In order to apply a circuit model, having both monomodal illumination and reception is necessary and important. We achieve this by using an array of structures with a periodicity less than one-half wavelength so that by diffraction theory, only a plane wave (i.e., a single mode) is transmitted. The array is made of essentially identical elements, so the collective response indeed provides us with the function of individual elements.

When the electric field is perpendicular to the NR array, the displacement current (dominant at optical frequencies), flows transversely across the NRs and air gaps as shown in Figs. 3(a) and 3(b). On the other hand, when the electric field is parallel to the NR array, the displacement current flows along both the NRs and air gaps [Figs. 3(c) and 3(d)]. The ratio of the optical potential to displacement current defines the optical lumped impedance of these nanostructures. These impedances are dependent on the shape and

TABLE I. Dimensions of the samples.

\begin{tabular}{lcllc}
\hline \hline Sample & Width $(w)$ & Gap $(g)$ & $w / g$ & Height $(h)$ \\
\hline A & $100 \mathrm{~nm}$ & $100 \mathrm{~nm}$ & 1 & $150 \mathrm{~nm}$ \\
B & $300 \mathrm{~nm}$ & $200 \mathrm{~nm}$ & 1.5 & $100 \mathrm{~nm}$ \\
$\mathrm{C}$ & $320 \mathrm{~nm}$ & $180 \mathrm{~nm}$ & 1.8 & $150 \mathrm{~nm}$ \\
$\mathrm{D}$ & $370 \mathrm{~nm}$ & $130 \mathrm{~nm}$ & 2.8 & $150 \mathrm{~nm}$ \\
E & $400 \mathrm{~nm}$ & $100 \mathrm{~nm}$ & 4 & $150 \mathrm{~nm}$ \\
\hline \hline
\end{tabular}



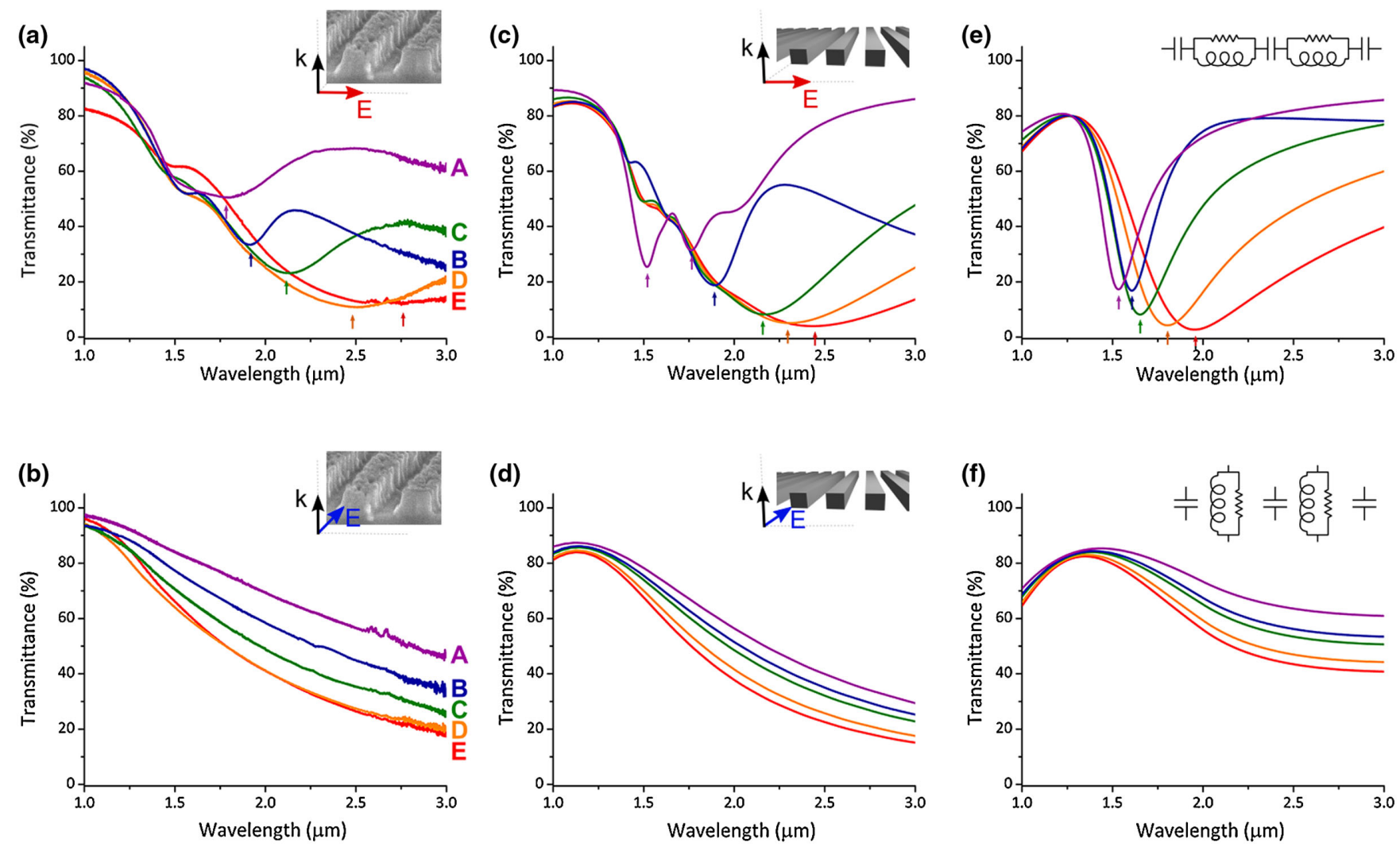

FIG. 2 (color online). Transmittance spectra of 5 different samples A through E. Experimentally measured data for (a) perpendicular (b) parallel polarization of the incident wave impinging on the NR arrays. Full-wave simulation results (c) and (d) and circuit theory calculation results (e) and (f), fully taking into account the effect of the substrate for two polarizations.

size of the structure, the constituent materials properties, the wavelength of the illumination, and the polarization of the illumination.

Indeed, we calculated these values for sample $\mathrm{E}$ when illuminated with light operating at a free-space wavelength of $2.5 \mu \mathrm{m}$. When the $E$ field is perpendicular to the NRs, the equivalent inductance and conductance of a single ITO NR are $0.396 \mathrm{nH} \mathrm{nm}$ and $7.8 \mu \mathrm{S} / \mathrm{nm}$, respectively. The equivalent capacitance of the air gap is $0.0133 \mathrm{aF} / \mathrm{nm}$. However, when the $E$ field is parallel to the NRs the impedance and conductance of the NR are changed as: $0.436 \mathrm{fH} / \mathrm{nm}$ and $1.25 \mathrm{~S} \mathrm{~nm}$, respectively. The capacitance of the air gap is calculated as $132.81 \mathrm{aF} \mathrm{nm}$.

We can determine the resonance conditions of these transmittance values using a simple circuit model. This enables a metatronic circuit designer to employ a forward design process and yields simple resonant conditions for series and parallel combinations of $\operatorname{Real}\left(\varepsilon_{L}\right) / \operatorname{Real}\left(\varepsilon_{c}\right)=$ $-(w / g)$ and $\operatorname{Real}\left(\varepsilon_{L}\right) / \operatorname{Real}\left(\varepsilon_{c}\right)=-(g / w)$, respectively, where in the present case $\varepsilon_{L}=\varepsilon_{\text {ITO }}$ and $\varepsilon_{C}=\varepsilon_{\text {air }}$.

We have demonstrated that by changing the circuit element values geometrically, we are able to shift the band-stop region. The variation of the resonant wavelength as a function of $w / g$ is shown in Fig. 3(e), using experimental measurements, full-wave simulations, and circuit theory calculations. The agreement among our experimental, numerical, and theoretical results is reasonably and functionally good. The difference in the circuit theory calculations arises since the dimensions of the NRs studied, while subwavelength, are not deeply subwavelength.
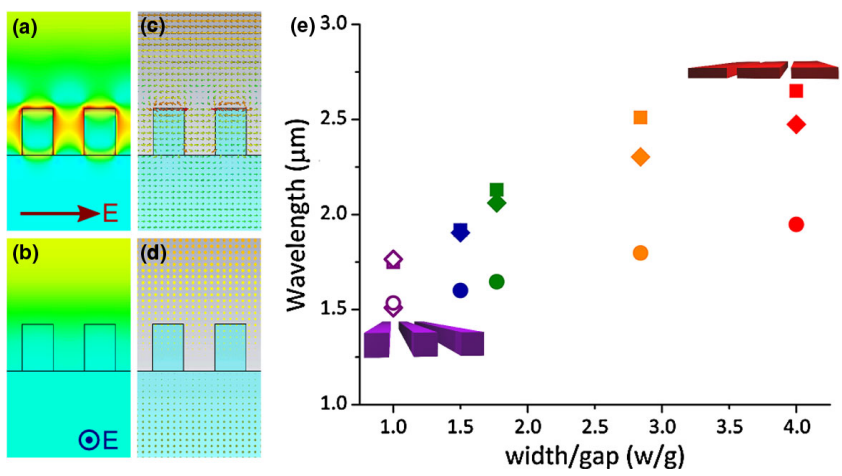

FIG. 3 (color online). Electric field profile of the sample ( $w=100 \mathrm{~nm}, g=100 \mathrm{~nm}$, and $h=150 \mathrm{~nm}$, sample A) for (a) perpendicular, and (b) parallel polarizations. Displacement current for (c) perpendicular and (d) parallel polarizations. (e) Comparison of the resonance wavelengths (band-stop wavelengths) of the transmittance for the five samples with the $E$ field perpendicular to the NRs (series combination). Note that for sample A with $w / g=1$, the full-wave numerical simulations result in two resonances. Square: Experimental data, Diamond: Full-wave simulation data and Circle: Circuit theory data. 
(a)

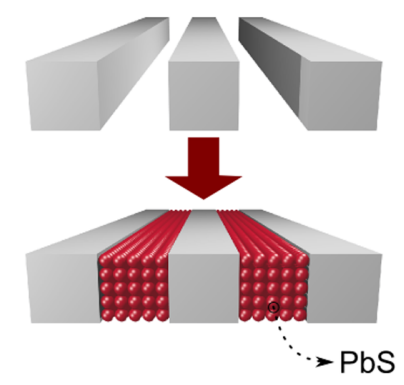

(d)

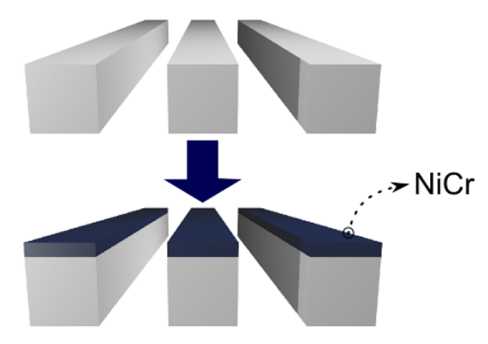

$100+($ (b)

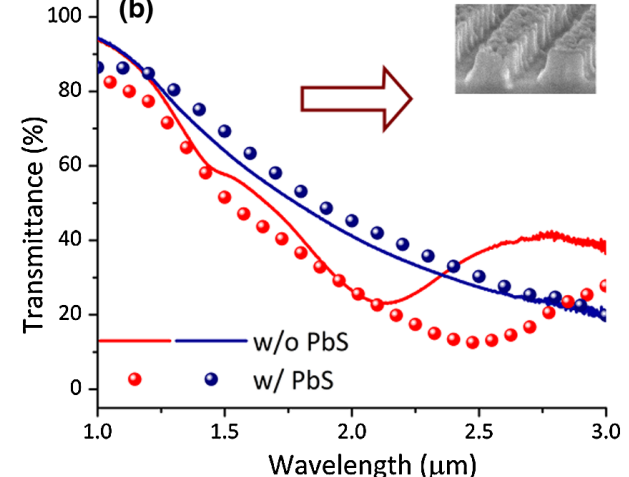

(e)

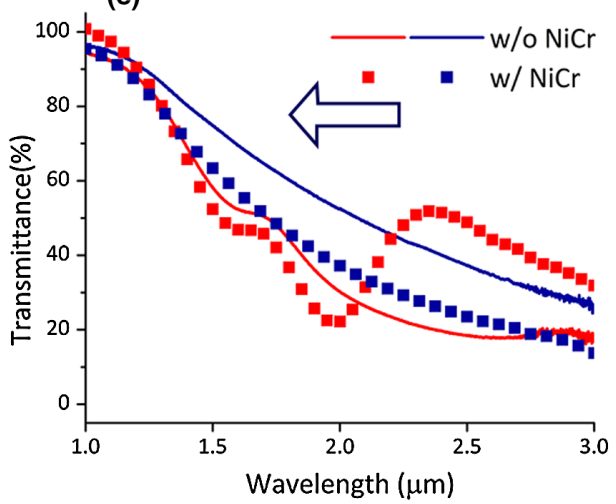

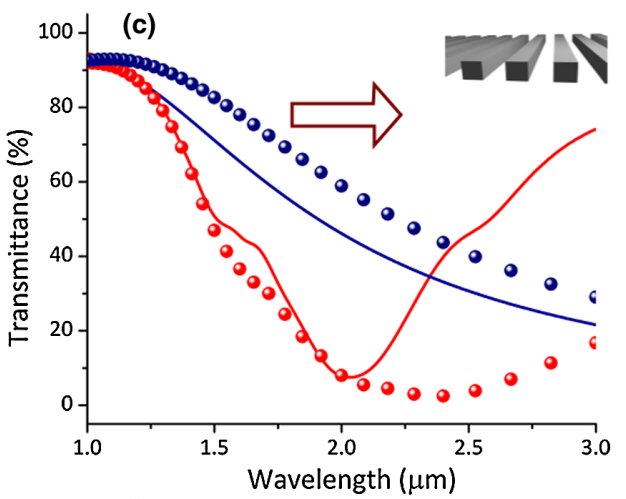

(f)

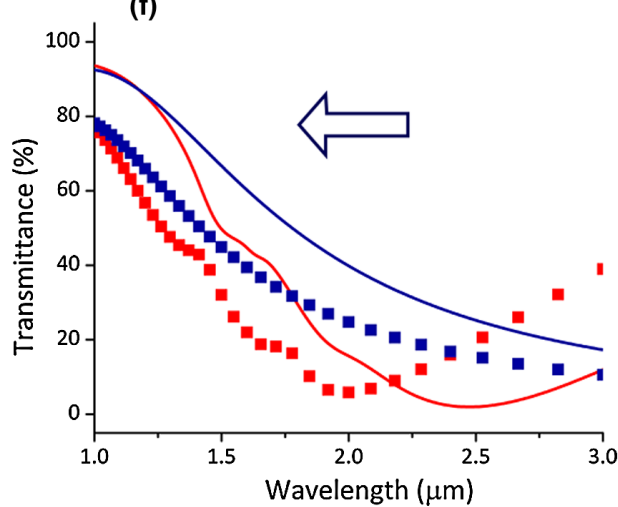

FIG. 4 (color online). Band-stop and bandpass regions were tailored by filling the air gaps of the ITO NR arrays $(w=320 \mathrm{~nm}$, $g=180 \mathrm{~nm}$ and $h=150 \mathrm{~nm}$, sample C) with PbS nanocrystals, as shown in (a) schematic and in (b) measured data, and (c) full-wave simulations. Red curves: $E$ field perpendicular to the NRs. Blue curves: $E$ field parallel to the NRs. Band-stop and bandpass regions were tailored by considering NiCr on top of the ITO NRs ( $w=400 \mathrm{~nm}, g=100 \mathrm{~nm}$, and $h=150 \mathrm{~nm}$, sample E) as shown in (d) schematic, with (e) measured data, and (f) full-wave simulation.

Nevertheless, the very elementary circuit formulas that are derived for quasistatic scenarios provide a simple design tool. The broader and redder resonances seen experimentally compared to numerically, are consistent with the inevitable imperfections in the nanofabrication processes including surface roughness, NR tapering, and the remaining (unetched) ITO. The agreement clearly shows the NR arrays function as filters consisting of arrays of nanocircuit elements and can be designed and modeled as circuits with responses in the NIR.

It must be emphasized that, it is in principle possible to cover from $1.5 \mu \mathrm{m}$ to around $3 \mu \mathrm{m}$ by changing the $w / g$ value. To demonstrate this, we have changed the $w / g$ in small steps using a single nanoimprint template and by changing the $\mathrm{O}_{2}$ etch time of the nanoimprint resist. From this single template we tailored the response, covering the spectrum from 2 to $3 \mu \mathrm{m}$ (see Supplemental Material, Fig. S2 [21]). For resonances shorter than $2 \mu \mathrm{m}$, we used a different nanoimprint template with smaller features.

A second approach to tailor the band-stop and bandpass region of the ITO NR filters is to add materials between the gaps or on top of the ITO NRs. Adding materials changes the inductance or capacitance value of the nanocircuit elements. First, we tune the filters by increasing the capacitance of the gap regions by filling them with high dielectric constant $\mathrm{PbS}$ nanocrystals [22] (sample F) as shown in Fig. 4(a). The optical properties $\mathrm{PbS}$ nanocrystals can be found in Supplemental Material, Fig. S1 [21]. The measured and simulated transmittance results for an ITO NR array in Figs. 4(b) and 4(c) show that the band-stop and bandpass regions are shifted towards longer wavelengths due to the higher capacitance value introduced by the nanocrystals compared to air. Second, in order to change the inductance value of the circuit elements, we consider the case where a $5 \mathrm{~nm}$ layer of $\mathrm{NiCr}$ is added on top of the ITO layer as shown in Fig. 4(d). This extra NiCr layer brings an additional inductance in parallel with that of ITO, resulting in a lowering of the equivalent inductance (since the inductances are considered to be in parallel) and thereby increasing the resonance frequency of the circuit. As a result, the operating wavelength shifts towards shorter wavelengths with the addition of this NiCr layer [Figs. 4(e) and 4(f)].

In summary, we have introduced NIR metatronic elements as building blocks for NIR filter metamaterials. We have fabricated arrays of NRs with rectangular cross sections made of ITO and tailored their optical response by engineering their impedance either through their crosssectional geometry or by adding materials. Using the equivalent circuit theory and FDTD simulations, we have demonstrated that we can design and analyze the nanoscale 
circuit element functionalities of such building blocks. This work opens up possibilities for 3D nanoscale filter cascades and tunable circuits as well as optical information processing at the nanoscale. Furthermore, the addition of phase change materials may be introduced in order to alter the impedance values of the circuit elements, enabling realtime control of the filter.

This work is supported by the U.S. Office of Naval Research Multidisciplinary University Research Initiatives (MURI) program Grant No. ONR-N00014-10-1-0942. The authors thank E.A. Gaulding and C.B. Murray of the University of Pennsylvania for providing the PbS nanocrystal sample. H. Caglayan and S.-H. Hong contributed equally to this work.

*Corresponding author. engheta@ee.upenn.edu

†Present address: Components \& Materials Research Laboratory, Electronics and Telecommunication Research Institute (ETRI), Daejeon 305-350, Republic of Korea.

[1] A. Yariv, Optical Electronics in Modern Communications (Oxford University, Oxford, 1997).

[2] W. L. Barnes, A. Dereux, and T.W. Ebbesen, Nature (London) 424, 824 (2003).

[3] E. Ozbay, Science 311, 189 (2006).

[4] T. W. Ebbesen, C. Genet, and S.I. Bozhevolnyi, Phys. Today 61, No. 5, 44 (2008).

[5] N. Engheta, A. Salandrino, and A. Alu, Phys. Rev. Lett. 95, 095504 (2005).

[6] N. Engheta, Science 317, 1698 (2007).

[7] A. Alu and N. Engheta, Phys. Rev. Lett. 103, 143902 (2009).
[8] M. G. Silveirinha, A. Alu, J. Li, and N. Engheta, J. Appl. Phys. 103, 064305 (2008).

[9] A. Alu, M. Young, and N. Engheta, Phys. Rev. B 77, 144107 (2008).

[10] H. Liu, Shivanand, and K.J. Webb, Phys. Rev. B 79, 094203 (2009).

[11] T. Baba and T. Ishihara, Phys. Status Solidi C 6, 327 (2009).

[12] G. V. Eleftheriades, Mater. Today 12, 30 (2009).

[13] K. Song and P. Mazumder, IEEE Trans. Nanotechnol. 8, 412 (2009).

[14] M. Staffaroni, J. Conway, S. Vedantam, J. Tang, and E. Yablonovitch, Photon. Nanostr. Fundam. Appl. 10, 166 (2012).

[15] Y. R. Padooru, A. B. Yakovlev, C. S. R. Kaipa, F. Medina, and F. Mesa, Phys. Rev. B 84, 035108 (2011).

[16] P.-Y. Chen and A. Alu, Phys. Rev. 82, 235405 (2010).

[17] Y. Sun, B. Edwards, A. Alu, and N. Engheta, Nat. Mater. 11, 208 (2012).

[18] P. R. West, S. Ishii, G. V. Naik, N. K. Emani, V.M. Shalaev, and A. Boltasseva, Laser Photon. Rev. 4, 795 (2010).

[19] A. Boltasseva and H. Atwater, Science 331, 290 (2011).

[20] H. S. Jeong, H.-J. Jeon, Yu. H. Kim, M. B. Oh, P. Kumar, S.-W. Kang, and H.-T. Jung, NPG Asia Mater. 4, e7 (2012).

[21] See Supplemental Material at http://link.aps.org/ supplemental/10.1103/PhysRevLett.111.073904 for details of the experimental and computational methods, materials dispersion, transmittance spectra for nanorods with a wide range of $w / g$, reflection spectra, and circuit theory taking into account substrate effects.

[22] M. A. Hines and G.D. Scholes, Adv. Mater. 15, 1844 (2003). 